\title{
Real-Time Exemplar-Based Face Sketch Synthesis
}

\author{
Yibing Song ${ }^{1}$, Linchao Bao ${ }^{1}$, Qingxiong Yang ${ }^{1 \star}$, and Ming-Hsuan Yang $^{2}$ \\ ${ }^{1}$ Department of Computer Science \\ Multimedia software Engineering Research Centre (MERC) \\ City University of Hong Kong, Hong Kong, China \\ MERC-Shenzhen, Guangdong, China \\ ${ }^{2}$ Department of Electrical Engineering and Computer Science \\ University of California at Merced
}

\begin{abstract}
This paper proposes a simple yet effective face sketch synthesis method. Similar to existing exemplar-based methods, a training dataset containing photosketch pairs is required, and a $K-\mathrm{NN}$ photo patch search is performed between a test photo and every training exemplar for sketch patch selection. Instead of using the Markov Random Field to optimize global sketch patch selection, this paper formulates face sketch synthesis as an image denoising problem which can be solved efficiently using the proposed method. Real-time performance can be obtained on a state-of-the-art GPU. Meanwhile quantitative evaluations on face sketch recognition and user study demonstrate the effectiveness of the proposed method. In addition, the proposed method can be directly extended to the temporal domain for consistent video sketch synthesis, which is of great importance in digital entertainment.
\end{abstract}

Keywords: Face Hallucination, Texture Synthesis.

\section{Introduction}

Face sketch synthesis has a wide range of applications ranging from digital entertainment to law enforcement. Regarding general sketch synthesis, there are basically two types of methods: the image-based method and the exemplar-based method. Imagebased sketch synthesis methods typically produce strokes and shadings according to edges in the input image while exemplar-based methods reconstruct new sketches from existing sketches. Although image-based methods can produce meaningful stylistic effects in some sense, their results are usually more like the input images, rather than artistic work from artists (since strokes only co-exist with strong edges in the image). Especially when it comes to face sketch, image-based methods will commonly fail to capture important facial details (see Fig. 1). On the other hand, exemplar-based methods can usually produce higher-quality sketches, but are computationally too intensive due to the matching process to a large amount of existing data.

The state-of-the-art exemplar-based face sketch synthesis methods are based on the Markov Random Field (MRF) models [19,22,24]. Generally, face photos and sketches

\footnotetext{
* Correspondence author. Complete experimental results (including the source code) are provided on the authors' webpage.
} 


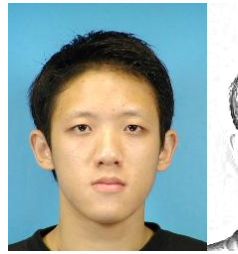

(a) Input

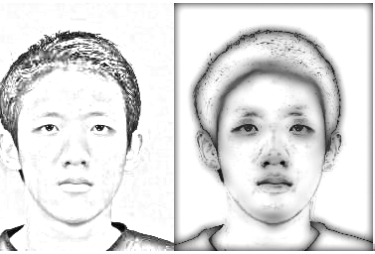

(b) $[1]$ (c) $[11]$

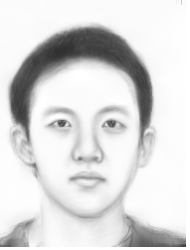

(d) Proposed

Fig. 1. Image-based and exemplar-based face sketch synthesis. (b) and (c) are sketches generated from image-based methods [1,11] and (d) is obtained from the proposed exemplar-based method. Image-based methods can handle general images well where image features can mainly be described through edges. For face photos where features can not be explicitly described as edges, these methods are less stylistic and cannot expressively present facial details compared with exemplar-based methods.

in training dataset are divided into patches, and the "best" sketch patch for representing a test photo patch will be selected via solving a MRF. These MRF-based methods can generally produce high-quality face sketches, but are computationally too intensive to become real-time applications. For example, the runtime of the most efficient exemplarbased method [24] for producing a $250 \times 200$ face sketch is over 40 seconds on a PC with Intel Core i7 $3.4 \mathrm{GHz}$ CPU.

In this work, we propose a face sketch synthesis method that can effectively produce face sketches in real-time speed. A $K$-NN search [3] is performed between a test photo and training photos to find the matched $K$ patches at each pixel location and displacement vectors ${ }^{1}$ are generated. Then the linear combination method [13] can be used at each pixel location to compute a corresponding sketch estimation. However, the resulting face sketch using this approach is noisy. An example is presented in Fig. 3(b).

The next step is to denoise the baseline performance which is the main contribution of this paper. The $K$ displacement vectors from each neighboring pixel around one center pixel are used to compute single sketch estimation using [13]. As a result, a simple averaging of these sketch estimations can greatly reduce noise and can be used as the final denoised sketch value (Fig. 3(f)).

The advantages of this work are summarized as follows:

- The proposed method is more efficient than the state-of-the-art in the literature. Real-time performance can be obtained on an NVIDIA Geforce GTX 780 GPU.

- Quantitative evaluation on face sketch recognition and user study demonstrate that the proposed method is more effective.

- The proposed method can be easily extended to temporal domain for consistent video sketch synthesis.

\footnotetext{
${ }^{1}$ The displacement vectors contain relative positions between test and training photo patches.
} 


\section{Related Work}

A literature survey of image-based and exemplar-based sketch synthesis methods is presented in the following:

\subsection{Image-based sketch synthesis methods}

There is huge amount of research work and commercial products on image-based sketch synthesis. Most of them utilize image edges to produce strokes or stylistic effects. For instance, bilateral weights which are responses to color edges are used in [11] to produce sketch images. Image tone and stroke structures are combined to complement each other in generating visually constrained results in [14]. As we mentioned before, image-based method will commonly fail to capture important facial details (as shown in Fig. 1), which would be sensitive to human beings.

\subsection{Exemplar-based sketch synthesis methods}

Research on exemplar-based methods derives from the supervised style transformation framework [12]. As illustrated in [10], exemplar-based methods outperform traditional parametric (image-based) methods because they can handle styles which are difficult to describe parametrically. Meanwhile their repertoire can be easily extended by the user at any time. Currently exemplar-based face sketch synthesis can be mainly categorized as profile sketch synthesis [7,21,5] and shading sketch synthesis $[16,19,24]$. Compared with profile sketches, shading sketches are more expressive in representing facial structures and thus more popular.

In [16] a global eigen-transformation is computed for synthesizing face sketches from face photos. A locally linear combination method is proposed in [13] to solve the non-linearity problems. A dense execution of [13] will lead to noise. The methods based on MRF models [19,22,24] alleviate noise when synthesizing sketches. The use of MRF encodes smoothness constraints on neighboring sketch patches during the global optimization process, thus artifacts between different sketch patches can be reduced. To bridge the modality difference between input images and output sketches, several algorithms $[23,18]$ consider the inter modality between photos and sketches by projecting facial features onto the same space.

\section{Face Sketch Synthesis}

We give a brief overview of the baseline algorithm in Sec. 3.1 and propose an image denoising method for face sketch synthesis in Sec. 3.2.

\subsection{Motivation}

The baseline approach of exemplar-based face sketch synthesis method [13] is illustrated in Fig. 2, which includes $K$-NN search and linear combination of patches specified in the following. If linear patch combination [13] is conducted densely, the synthesized 


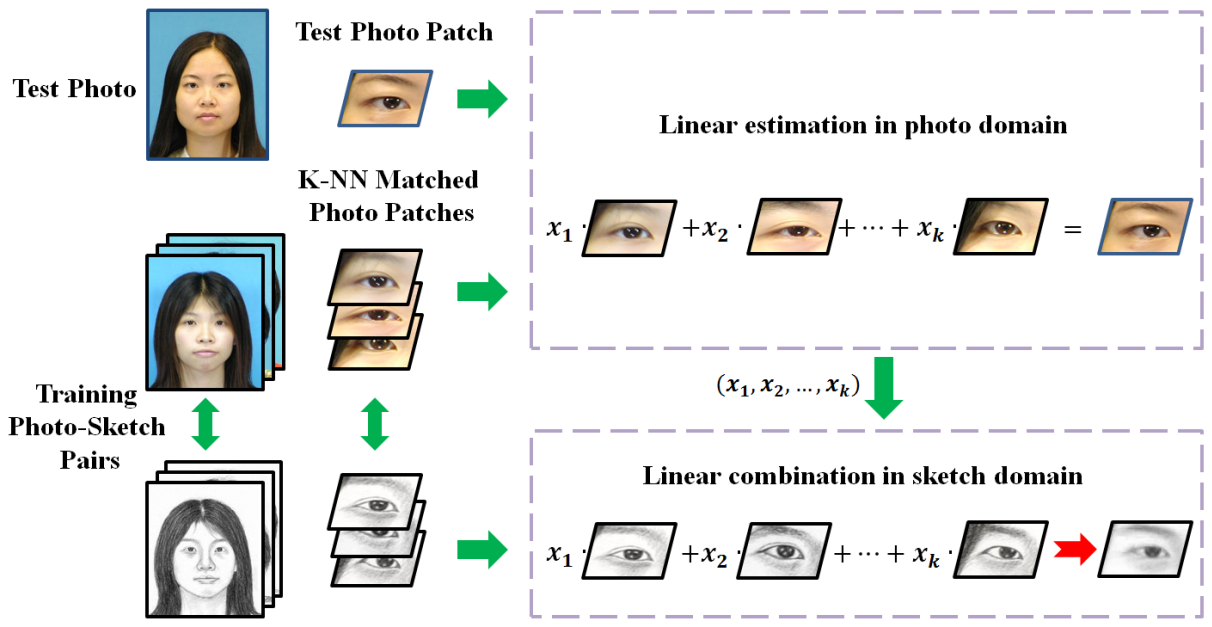

Fig. 2. Illustration of the baseline approach [13]. For each patch in the test photo, $K$-NN search and linear estimation are performed in the photo domain to obtain the matched training photo patches and linear weights $\left(x_{1}, x_{2}, \cdots, x_{k}\right)$. The weights are used in the sketch domain to map the corresponding training sketch patches into output patch.

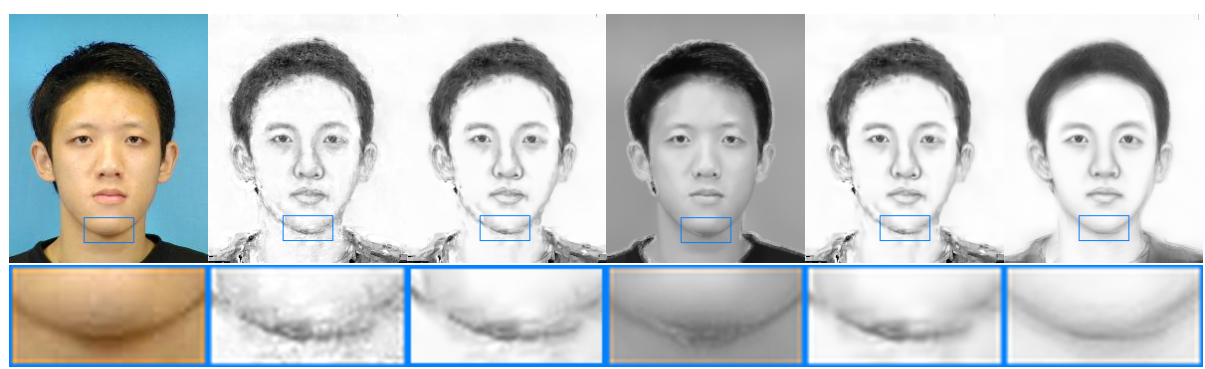

(a)Input (b)Base [13] (c)NLM [6] (d)Joint NLM (e)BM3D [8] (f)Proposed

Fig. 3. Baseline synthesized results and improvements. (a) is an input test photo and (b) is the sketch obtained from the baseline approach [13]. (c) to (e) are denoised sketches obtained from NLM [6], a simple extension of NLM with (a) as the guidance, and BM3D [8], respectively. (f) is the sketch obtained from proposed sketch denoising algorithm.

sketch (Fig. 3(b)) contains significant amount of noise due to the modality gap between photo and sketch.

A potential solution is using existing image denoising algorithms to eliminate the sketch noise. However, a direct use of the state-of-the art denoising algorithms, e.g., NLM [6] and BM3D [8] shows little improvement as can be seen in Fig. 3(c) and (e). A simple extension is using the test photo in Fig. 3(a) as the guidance image for NLM. 
The denoised sketch, however, looks similar to photo rather than sketch (Fig. 3(d)). We thus aim at developing a denoising algorithm that is suitable for face sketch synthesis.

$K$-NN Search and Linear Estimation For the patch centered at each pixel in a test photo, we search for the most similar patch on each training photo and the top $K$ similar patches are selected. When performing $K-\mathrm{NN}$ search we generate a cost volume by continuously shift training photos to compute a difference image slice with respect to test photo. Then box filer [17] is applied to the cost volume for filtering (the filtered cost is the patch matched cost). Let $\mathbf{T}_{p}$ denote a vector containing the pixel values of the patch centered at pixel $p$ in the tested photo, and $\mathbf{I}_{p}^{1}$ to $\mathbf{I}_{p}^{K}$ denote vectors containing the pixel values of the $K$ corresponding matched patches, respectively. The coefficients of a linear mapping function that maps the $K$ matched patches to the testing patch $\mathbf{T}_{p}$ can be obtained by solving the following linear system

$$
x_{p}^{1} \mathbf{I}_{p}^{1}+x_{p}^{2} \mathbf{I}_{p}^{2}+\cdots+x_{p}^{K} \mathbf{I}_{p}^{K}=\mathbf{T}_{p},
$$

where $x_{p}^{1}$ to $x_{p}^{K}$ are the coefficients. When $K$ is low ( $K=5$ in this paper), it is a lowrank system and the coefficients can be computed efficiently using a conjugate gradient solver [15].

\subsection{Sketch Denoising}

In Sec. 3.1, $K$ displacement vectors $\Delta_{p}=\left[\Delta_{p}^{1}, \Delta_{p}^{2}, \cdots, \Delta_{p}^{K}\right]$ are computed and the corresponding linear mapping functions are represented by coefficients $X_{p}=\left\{x_{p}^{1}, x_{p}^{2}, \cdots, x_{p}^{K}\right\}$ at each pixel location $p$. Let $S$ denote the training sketch images so that $S_{p+\Delta_{p}^{1}}$ to $S_{p+\Delta_{p}^{K}}$ correspond to the sketch values selected by the $K-\mathrm{NN}$ search at pixel $p$ in Sec. 3.1. A coarse sketch estimation at each pixel $p$ can be obtained to form a coarse sketch image as follows:

$$
E_{p}=\sum_{k=1}^{K} x_{p}^{k} S_{p+\Delta_{p}^{k}} .
$$

A direct use of image denoising method like NLM [6] for sketch denoising can be presented as weighted sum over the coarse sketch image:

$$
S_{p}^{N L M}=\frac{\sum_{q \in \Psi_{p}} w(p, q) E_{q}}{\sum_{q \in \Psi_{p}} w(p, q)},
$$

where $\Psi_{p}$ is a local patch centered at pixel $p$ and the weight

$$
w(p, q)=\exp \left(-\frac{d\left(\psi_{p}, \psi_{q}\right)}{2 \sigma^{2}}\right)
$$

depends on the distance $d\left(\psi_{p}, \psi_{q}\right)$ between $\psi_{p}$ and $\psi_{q}$ which are two relatively smaller sketch patches centered at pixel $p$ and $q$, respectively. This type of direct application of image denoising techniques is not suitable for noisy sketch image as shown in Fig. 3(c) and (e). A simple extension is to adjust the weight by extracting the two local patches 


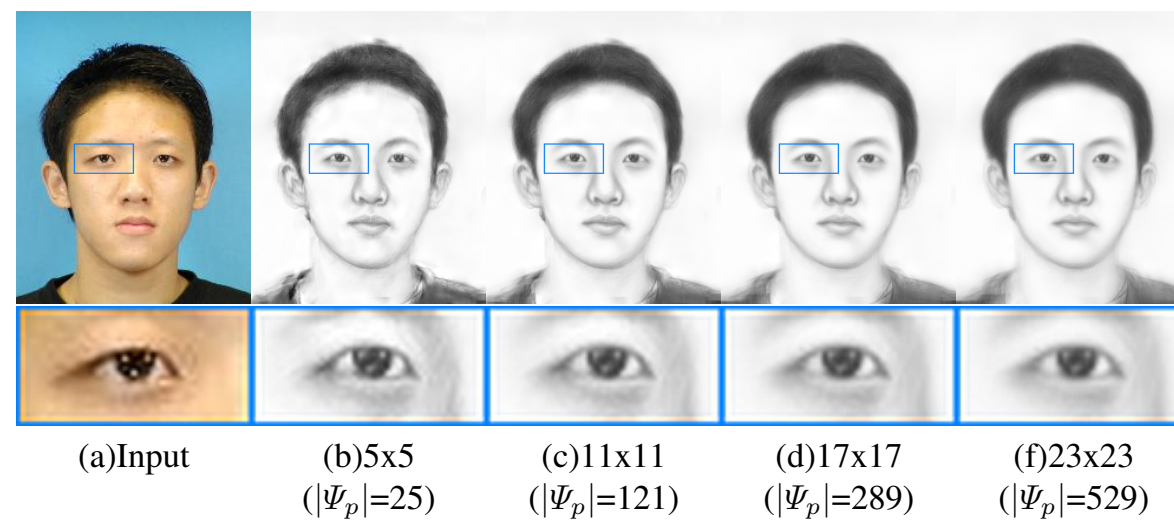

Fig. 4. Performance with respect to the size of patch $\Psi_{p}$. The proposed SSD method appears to be fairly robust against different values of $\left|\Psi_{p}\right|$ when it is sufficient large, e.g., 100) to eliminate the sketch noise. It well preserves facial details like the tiny eye reflections.

$\psi_{p}$ and $\psi_{q}$ from the noise-free photos to eliminate the errors due to sketch noise. That is, we use the noise-free photo as a guidance to compute the weight at each pixel location. However, the improvement is not nontrivial as shown in Fig. 3(d).

The reason that traditional image denoising methods and their simple extensions fail lies in the use of incorrect weights. The coarse sketch image is not natural, and the traditional assumption that two image pixels are similar if the two corresponding patches are similar is invalid for a coarse sketch image.

Instead of determining a better representation of the weight $w(p, q)$ to represent the similarity between any two sketch image pixels, we focus on grouping sketch estimates that are believed to be similar. This idea is motivated by the BM3D [8] method which groups correlated patches in the image domain to create multiple estimations.

The grouping scheme used in this paper is based on linear local model. Under this assumption the linear mapping function computed at a pixel $q$ from $K-\mathrm{NN}$ can be used together with the displacement vectors to compute not only the sketch estimate at pixel $q$ but also the sketch estimates for all pixel $p$ insides a local neighbor of pixel $q$. Let $E_{p}^{q}$ denote the sketch estimate at pixel $p$ that are computed based on the linear mapping function and displacement vectors obtained using $K-\mathrm{NN}$ at pixel $q$, we have

$$
E_{p}^{q}=\sum_{k=1}^{K} x_{q}^{k} S_{p+\Delta_{q}^{k}}
$$

where $E_{p}^{p}=E_{p}$ in Eq. 2. The sketch estimate $E_{p}^{q}$ is guaranteed to be similar to $E_{p}$ according to the linear local model, as long as pixel $p$ and $q$ are close. The denoising process is thus straightforward. Let $S^{\mathrm{SSD}}$ denote the denoised sketch image, the denoised sketch value at a pixel location $p$ is

$$
S_{p}^{\mathrm{SSD}}=\frac{\sum_{q \in \Psi_{p}} 1 \cdot E_{p}^{q}}{\sum_{q \in \Psi_{p}} 1}=\frac{1}{\left|\Psi_{p}\right|} \sum_{q \in \Psi_{p}} E_{p}^{q},
$$




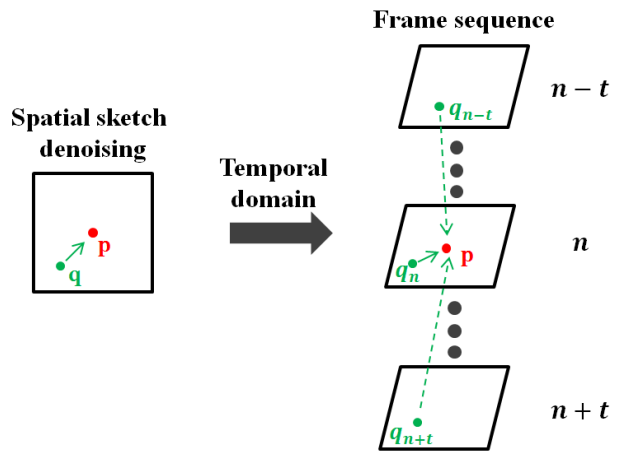

Fig. 5. The SSD method can be extended to the temporal domain. In SSD, pixel $p$ can only receives estimation from neighboring pixel in $\Psi_{p}$. In TSD, pixel $p$ in the $n$-th frame receives estimation from neighboring pixel in $\Psi_{p}$ in a number of frames within a time window.

where $\left|\Psi_{p}\right|$ is the number of pixels in patch $\Psi_{p}$. We refer to this sketch denoising method as Spatial Sketch Denoising (SSD) and extend it to the time domain in the following. The size of patch $\left|\Psi_{p}\right|$ is the only parameter used in the proposed SSD algorithm. However, the performance of SSD is robust to this parameter as demonstrated in Fig. 4. Specifically the proposed SSD method can well preserve facial details like the tiny eye reflections in the closeups.

Temporal Sketch Denoising (TSD) The proposed SSD method can be extended to the temporal domain for video sketch synthesis. Temporal incoherence of synthesized results lead to noticeable artifacts in videos. As shown in Fig. 5, pixels on the synthesized sketch can be considered as the averaging result of multiple estimations created from neighboring pixels from both spatial and temporal domains. Let $t$ denote temporal radius in the time window, the denoised sketch value at pixel $p$ for the $n$-th frame is

$$
S_{p}^{\mathrm{TSD}}=\frac{1}{(2 t+1)\left|\Psi_{p}\right|} \sum_{i=n-t}^{n+t} \sum_{q_{i} \in \Psi_{p}} E_{p}^{q_{i}},
$$

where $E_{p}^{q_{i}}$ is the sketch estimation computed for pixel $p$ based on the linear mapping function and displacement vectors from pixel $q_{i}$ in the $i$-th frame.

\section{Experiments}

In all the experiments, we set the search radius of displacement vector to 5 pixels in the $K$-NN search process (i.e., $|\Delta|=121$ ). The number of candidate correspondences $K$ is set to 5 . The size of the $\Psi$ is $17 \times 17$ pixels. We conduct quantitative and qualitative evaluations in two aspects. First, we evaluate the proposed algorithm against state-of-the-art methods on benchmark datasets in terms of effectiveness and run time performance. Second, we evaluate algorithms on generating user preferred sketches with human subject studies. 

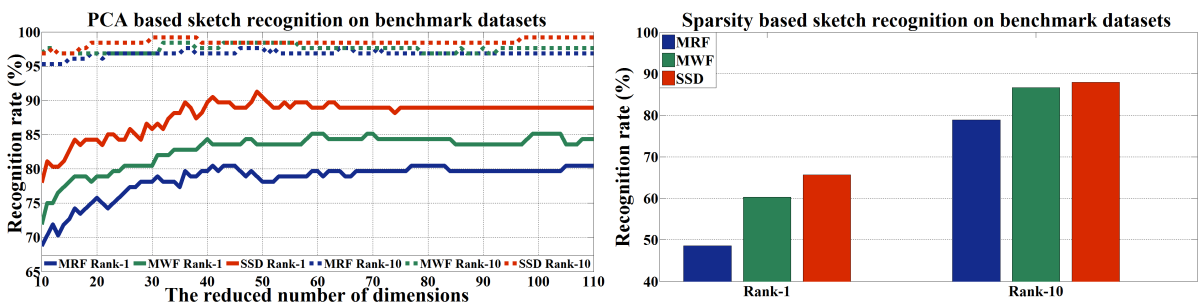

Fig. 6. Quantitative evaluations on benchmark datasets. The proposed SSD method performs favorably against the MRF and MWF methods under PCA based [9] and sparsity based [20] sketch recognition rates.

\subsection{Evaluations on Benchmark Datasets}

We carry out quantitative and qualitative experiments on the CUHK student dataset [19] and the AR dataset [2]. The CUHK student dataset [19] consists of 188 photo-sketch pairs where 88 pairs are used for training and the rest are for testing. In the AR dataset [2] which consists of 123 photo-sketch pairs, we use the leave-one-out strategy as conducted in the MRF and MWF methods. The proposed SSD method takes about 4 seconds to process one test photo on the CUHK student dataset using $3.4 \mathrm{GHz}$ i 7 machine and $\mathrm{C}++$ programming language. In addition, the proposed method can be executed in 20 FPS on an Nvidia GeForce GTX 780 GPU, which is $80 \times$ faster than CPU implementation. Moreover, the proposed algorithm does not need any off-line training whereas the other methods [19,22,24,23,18] entail such procedures.

Quantitative evaluation of face sketch synthesis methods can be conducted through face sketch recognition as suggested in [22]. That is, for each photo in the training set, the synthesized sketch should be matched to the corresponding sketch drawn by an artist. If an algorithm achieves higher sketch recognition rates, it suggests that this method is more effective to synthesize sketches.

We perform sketch recognition evaluation on the whole 223 synthesized sketches from the CUHK [19] and AR [2] datasets. The proposed SSD method is evaluated with the state-of-the-art MRF and MWF methods using two metrics: PCA based recognition rate [9] and sparsity based recognition rate [20]. The rank-1 and rank-10 sketch recognition rates are also used for evaluation.

Sketch recognition results of different methods are shown in Fig. 6. The PCA based and sparsity based sketch recognition rates indicate the proposed SSD method achieves higher recognition rates than those with the MRF and MWF methods under the rank1 metric. Using the rank-10 metric, the proposed SSD method performs well against the state-of-the-art methods in both PCA and sparsity based recognition rates. Fig. 6 demonstrates the proposed SSD method performs favorably against the MRF and MWF methods in effectiveness to synthesize sketches on average.

Qualitative evaluation results of the SSD, MRF and MWF methods are shown in Fig. 7. The holistic results show that the MRF method does not synthesize dominant facial structures (i.e., eyes or hair) well. Without linear estimation the MRF method is 



$\begin{array}{lll}\text { (a) Test photo (b) MRF [19] (c) MWF [24] } & \text { (d) SSD }\end{array}$

Fig. 7. Qualitative evaluation on benchmark datasets. (a) test photos. (b) sketches generated by the MRF method. (c) sketches generated by the MWF method. (d) sketches generated by the proposed SSD method. The results show that the proposed SSD method performs favorably against the MRF and MWF methods in preserving details and removing noise.

not effective in synthesizing a suitable patch from training data for some test patches with facial features significantly different from those of the training set. On the other 


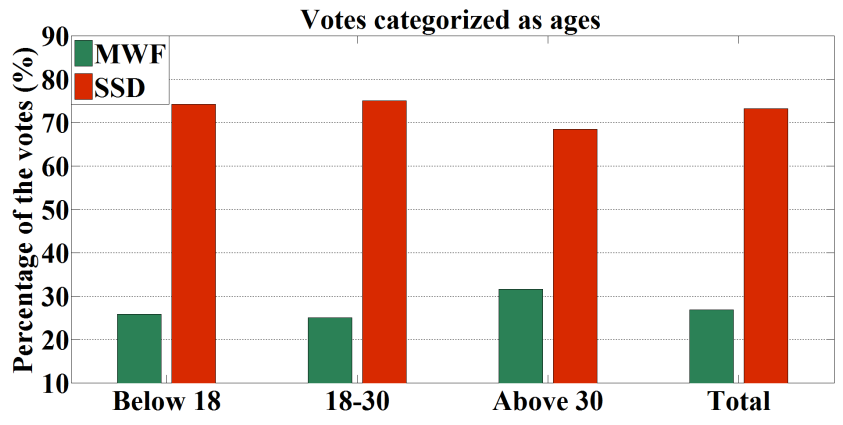

Fig. 8. Quantitative evaluation of user study on the real-world photos. There were 153 subjects participating. Among them 31 people are under age 18, 84 people age from 18 to 30, and 38 people are above age 30 . Their sketches are synthesized by using the CUHK student dataset. The proposed SSD method performs favorably against the MWF method across all demographics.

hand, the results from the MWF method contain significant amount of noise on some facial components (i.e., noses and mouths). Details of some dominant facial regions are also blurred (i.e., hair and mouths). With the linear estimation process and sketch denoising, the proposed SSD method is able to synthesize distinct features and reduce noise. More qualitative evaluations can be found in the complete experimental results.

\subsection{Evaluations on Real-World Datasets}

In this section, we conduct evaluations on real-world photos and videos. In Sec. 4.1 the MRF method is shown not effective to synthesize facial structures. Thus the focus is put on the evaluation of the proposed SSD, TSD and MWF methods.

Evaluations on Real-World Photos For the face photos captured in the real-world scenes we first rectify them to fit the benchmark dataset condition. We then perform a user study to find which kind of sketches is user preferred. The criteria of sketch evaluation is subjective and in some cases they are the opposite (e.g., some prefer sketches to be similar to photos for realism while others opt for stylistic sketches). Thus, user preference is achieved by letting subjects evaluate synthesized sketches of their own photos.

There are 153 participants in the user study. For each test, we synthesize two sketches of one subject by using the proposed SSD and MWF methods. Then the subject is asked to identify the preferred one according to his (her) own preference. The answer of each subject is considered as one vote of each method. Fig. 8 shows the final votes of each method where the proposed SSD method performs favorably against the MWF method across all demographics.

Fig. 9 shows some results from the user study. On each row, the sketches marked in red are the user preferred sketches. On the top two rows there is significant noise which deteriorates the facial details (e.g., eyes and noses) shown in (b) while this noise 


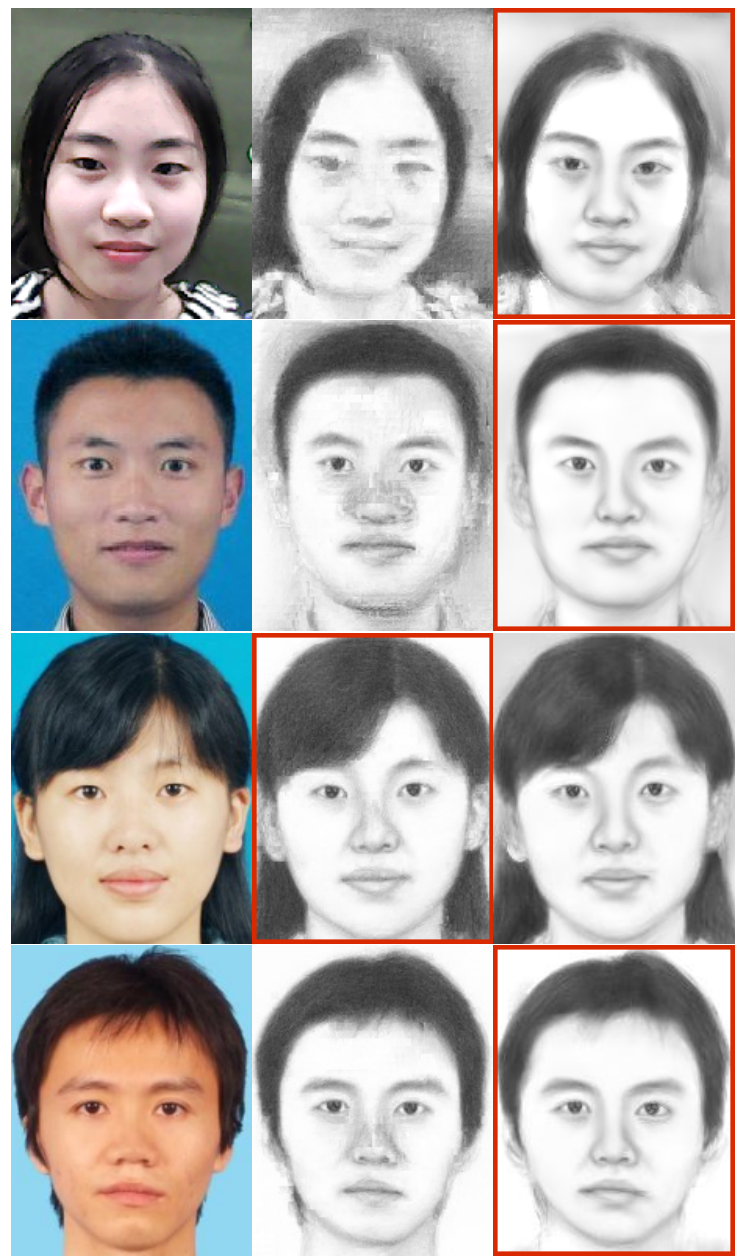

$\begin{array}{lll}\text { (a) Test photo } & \text { (b) MWF [24] } & \text { (c) SSD }\end{array}$

Fig. 9. Qualitative evaluation of user study on real-world photos. (a) test photos. (b) sketches generated by MWF. (c) sketches generated by SSD. The sketches marked in red indicate user preference. In the top two rows significant noise deteriorates the facial details of sketches (e.g., eyes and noses) shown in (b). So the majority of subjects do not prefer. In the bottom two rows the facial details of sketches are effectively synthesized in both (b) and (c). User preference is random.

is greatly reduced in (c). It indicates when the facial structures of sketches can not be synthesized effectively the majority of subjects do not prefer. On the bottom two rows the facial details are effectively synthesized in both (b) and (c). Thus, user preference is random and there is almost no difference between the votes of these two methods in this case. As in the real-world datasets significant noise often occurs and deteriorates 


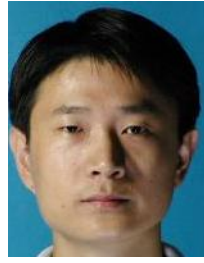

(a) Test photo

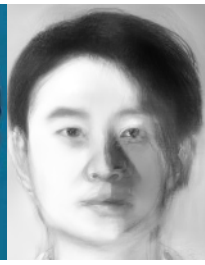

(b) SSD

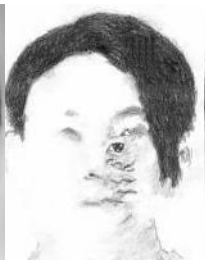

(c) MRF [19] (d) MWF [24]

Fig. 10. Limitations of sketch synthesis methods. (a) test photo captured with cast shadow on the right. (b)-(d) are synthesized results by the proposed SSD, MRF and MWF methods, respectively. Incorrect patch matching occurs in all the methods. For (c) and (d), significant noise is generated on the facial parts where the lighting conditions are different from those in the training dataset. In (b) blurry artifacts occur on those facial parts.

the facial details of the sketches synthesized by the MWF method while noise is greatly reduced on the ones synthesized by the SSD method, the proposed SSD method performs favorably against the MWF method on average across all demographics. Fig. 9 demonstrate that the proposed SSD method is more effective in reducing noise and thus synthesizing user preferred sketches.

Evaluations on the Real-World Videos For video sketch synthesis we evaluate the proposed TSD method with the SSD and MWF methods. For the TSD method we set the number of temporal coherent frames as 5. As the temporal coherence can not be displayed clearly on the paper, a video is included in the complete experimental results to demonstrate the temporal consistency of the proposed TSD method.

\section{Limitations and Discussion}

While the proposed algorithm performs well in synthesizing face sketches, the performance hinges on whether good patches can be matched from the training set to a test photo. When lighting conditions are significantly different from those in the dataset, incorrect patch matching occurs as a result of inaccurate $K$-NN search. As shown in Fig. 10(a), a test photo is captured with cast shadow on the right. For the face regions where patches can be found in the training set with similar lighting conditions (e.g., left cheek), the SSD, MRF and MWF methods perform normally. However, none of these methods perform well when this assumption does not hold.

Insufficient search range may also lead to incorrect patch matching. However, patch match within a large search radius inadvertently increase the computational load significantly. Nevertheless, this problem can be alleviated with efficient algorithms to search for patches such as the PatchMatch (PM) [4] method. Fig. 11 shows some experiments with photos of varying poses. The synthesized results in Fig. 11(b) and (d) are generated from the SSD and MWF methods using the same search range adopted on the benchmark datasets. When patches can be correctly matched across different poses by 


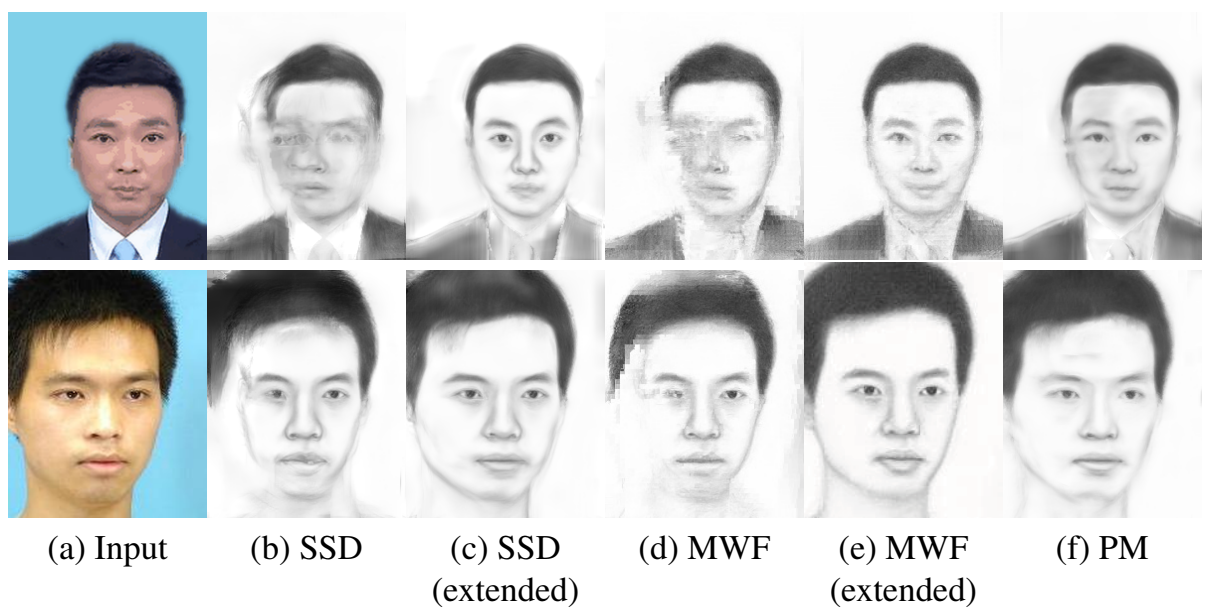

Fig. 11. Integration of PatchMatch [4]. (a) input photo with varying pose. (b) sketch generated by the SSD method using original search range. (c) sketch generated by the SSD method using large search range. (d) sketches generated by the MWF method original search range. (e) sketch generated by the MWF method using large search range. (f) sketch generated by the integration of PatchMatch and SSD method, which takes constant time cost regardless of search range.

increasing the search range, better sketches can be generated as shown in (c) and (e) at the expense of significant computational loads. Fig. 11(f) shows the results by integrating the SSD algorithm and the PatchMatch method without increasing computational load significantly.

\section{Concluding Remarks}

This paper formulates face sketch synthesis as an image denoising problem. The modality gap between photos and sketches in the training dataset leads to noise on the baseline results. State-of-the-art MRF based methods can be formulated as the baseline improvements by adding smoothness constraints to reduce noise when synthesizing sketch patches. Instead of involving MRF framework, a simple yet effective sketch denoising method is proposed from the perspective of image denoising. The proposed method is more efficient and achieves real-time performance on GPU. Quantitative evaluations on face sketch recognition and user study demonstrate the effectiveness of the proposed algorithm for synthesizing face sketches. Furthermore, the proposed method can be easily extended to the temporal domain for video sketch synthesis.

\section{Acknowledgement}

This work was supported in part by a GRF grant from the Research Grants Council of Hong Kong (RGC Reference: CityU 122212), the NSF CAREER Grant \#1149783 and 
NSF IIS Grant \#1152576. The authors would like to thank Hao Zhou for providing his code for [24].

\section{References}

1. Adobe: Adobe photoshop cs6

2. Aleix, M., Robert, B.: The ar face database. Tech. Rep. CVC Technical Report 24, Purdue University (1998)

3. Altman, N.S.: An introduction to kernel and nearest-neighbor nonparametric regression. The American Statistician (1992)

4. Barnes, C., Shechtman, E., Finkelstein, A., Goldman, D.: Patchmatch: a randomized correspondence algorithm for structural image editing. SIGGRAPH (2009)

5. Berger, I., Shamir, A., Mahler, M., Carter, E., Hodgins, J.: Style and abstraction in portrait sketching. SIGGRAPH (2013)

6. Buades, A., Coll, B., Morel, J.M.: A non-local algorithm for image denoising. In: CVPR (2005)

7. Chen, H., Xu, Y.Q., Shum, H.Y., Zhu, S.C., Zheng, N.N.: Example-based facial sketch generation with non-parametric sampling. In: ICCV (2001)

8. Dabov, K., Foi, A., Katkovnik, V., Egiazarian, K.: Image denoising by sparse 3-d transformdomain collaborative filtering. TIP (2007)

9. Delac, K., Grgic, M., Grgic, S.: Independent comparative study of pca, ica, and lda on the feret data set. IJIST (2005)

10. Freeman, W.T., Tenenbaum, J.B., Pasztor, E.: An example-based approach to style translation for line drawings. MERL Technical Report (1999)

11. Gastal, E.S., Oliveira, M.M.: Domain transform for edge-aware image and video processing. SIGGRAPH (2011)

12. Hertzmann, A., Jacobs, C.E., Oliver, N., Curless, B., Salesin, D.H.: Image analogies. SIGGRAPH (2001)

13. Liu, Q., Tang, X., Jin, H., Lu, H., Ma, S.: A nonlinear approach for face sketch synthesis and recognition. In: CVPR (2005)

14. Lu, C., Xu, L., Jia, J.: Combining sketch and tone for pencil drawing production. In: NPAR (2012)

15. Paige, C.C., Saunders, M.A.: Lsqr: An algorithm for sparse linear equations and sparse least squares. ACM Transactions on Mathematical Software (1982)

16. Tang, X., Wang, X.: Face sketch synthesis and recognition. In: CVPR (2003)

17. Viola, P., Jones, M.: Rapid object detection using a boosted cascade of simple features. In: CVPR (2001)

18. Wang, S., Zhang, L., Y., L., Pan, Q.: Semi-coupled dictionary learning with applications in image super-resolution and photo-sketch synthesis. In: CVPR (2012)

19. Wang, X., Tang, X.: Face photo-sketch synthesis and recognition. PAMI (2009)

20. Wright, J., Yang, A.Y., Ganesh, A., Sastry, S.S., Ma, Y.: Robust face recognition via sparse representation. PAMI (2009)

21. Xu, Z., Chen, H., Zhu, S.C., Luo, J.: A hierarchical compositional model for face representation and sketching. PAMI (2008)

22. Zhang, W., Wang, X., Tang, X.: Lighting and pose robust face sketch synthesis. In: ECCV (2010)

23. Zhang, W., Wang, X., Tang, X.: Coupled information-theoretic encoding for face photosketch recognition. In: CVPR (2011)

24. Zhou, H., Kuang, Z., Wong, K.: Markov weight fields for face sketch synthesis. In: CVPR (2012) 